\title{
An eye-tracking method to reveal the link between gazing patterns and pragmatic abilities in high functioning autism spectrum disorders
}

\author{
Ouriel Grynszpan ${ }^{1 *}$ and Jacqueline Nadel $^{2}$ \\ ${ }^{1}$ Institut des Systèmes Intelligents et de Robotique (ISIR), Université Pierre et Marie Curie, Centre National de la Recherche Scientifique, Paris, France \\ ${ }^{2}$ Centre Emotion, Hôpital de La Salpêtrière, Paris, France
}

\section{Edited by:}

John J. Foxe, Albert Einstein

College of Medicine, USA

\section{Reviewed by:}

Hans-Peter Frey, Albert Einstein

College of Medicine, USA

Julia Irwin, Haskins Laboratories,

USA

Karri Gillespie-Smith, University of

West of Scotland, UK

\section{*Correspondence:}

Ouriel Grynszpan, Institut des Systèmes Intelligents et de

Robotique (ISIR), Université Pierre et Marie Curie, Centre National de

la Recherche Scientifique, Pyramide - T55/65, CC 173 - 4 place Jussieu,

75005 Paris, France

e-mail: ouriel.grynszpan@upmc.fr
The present study illustrates the potential advantages of an eye-tracking method for exploring the association between visual scanning of faces and inferences of mental states. Participants watched short videos involving social interactions and had to explain what they had seen. The number of cognition verbs (e.g., think, believe, know) in their answers were counted. Given the possible use of peripheral vision that could confound eye-tracking measures, we added a condition using a gaze-contingent viewing window: the entire visual display is blurred, expect for an area that moves with the participant's gaze. Eleven typical adults and eleven high functioning adults with Autism Spectrum Disorders (ASD) were recruited. The condition employing the viewing window yielded strong correlations between the average duration of fixations, the ratio of cognition verbs and standard measures of social disabilities.

Keywords: eye-tracking, gaze-contingent display, facial expressions, theory of mind, cognition verbs

\section{INTRODUCTION}

The rise of affective computing during the last two decades has offered a wide range of possibilities for designing new tools that foster the study of social and emotional impairments. Affective computing is a stream of research that strives to empower computers with abilities to detect, process and respond to social and emotional signals emitted by human users (Picard, 2000). These advances appear to be especially relevant to Autism Spectrum Disorders (ASD) where social interactions represent the core deficit of the syndrome. The last decade has witnessed a steep increase in the number of projects devoted to innovative technologies for evaluating, assisting or training individuals with ASD (Grynszpan et al., 2014). Besides, a promising trend of research relies on the use of eye-tracking to gain a deeper understanding of the cognitive processes that characterize ASD (Boraston and Blakemore, 2007). The exploratory study reported here seeks to present an eye-tracking method for examining social processing in ASD, which takes advantage of real-time eye-based computer interaction.

Social misunderstanding in ASD has been linked to atypical visual scanning patterns (Klin et al., 2002a). Earlier eye-tracking studies used static images to examine the dysfunctional visual exploration of faces attributed to ASD (Pelphrey et al., 2002; van der Geest et al., 2002; Dalton et al., 2005; Corden et al., 2008; Fletcher-Watson et al., 2009). The development of eyetracking technology has offered new opportunities to investigate gaze through the use of dynamic social scenes, which are closer to real life settings (Klin et al., 2002a). This stream of research has raised hopes that eye-tracking measures would help identify behavioral markers and enable refining the autistic symptomatology. Yet, studies reported inconsistent results: Klin et al's (2002b) initial report that adolescents and adults tended to focus more on the mouth and less on the eyes than typical controls has only been partly confirmed (Speer et al., 2007; Norbury et al., 2009). Jones et al. (2008) showed that fixation times on the eyes were reduced in toddlers with ASD, but more recent studies failed to reproduce this finding (Nakano et al., 2010; Chawarska et al., 2013). The most consistent discriminating measure between ASD and typical participants appears to be the fixation times on faces (Riby and Hancock, 2009; von Hofsten et al., 2009; Grynszpan et al., 2012a; Rice et al., 2012; Chawarska et al., 2013; Magrelli et al., 2013). Another promising line of research has been to examine the association between gaze data and standard evaluation instruments of ASD. Klin et al. (2002b) reported that the time spent looking at the mouth region correlated with social abilities in high functioning adolescents and adults. Later research suggested that this correlation depended on the characteristics of the video material, such as the number of characters displayed (Speer et al., 2007) or the space taken on the screen by human faces (Rice et al., 2012). Norbury et al. (2009) failed to reproduce this outcome, but found a negative correlation between the fixation times on the eyes and 
communicative competencies. The observation that verbally able adults with ASD showed a high association between fixations on facial features and social or communicative abilities has led to the hypothesis that their visual scanning strategies was connected to their linguistic proficiency (Klin et al., 2002b; Norbury et al., 2009).

Studies on linguistic competencies in ASD reveal a primary deficit in pragmatics (Tager-Flusberg, 2000). Grynszpan et al. (2008) suggest that children with ASD experience difficulties in using facial expressions as cues to resolve pragmatic ambiguities in dialogs. Their poor performance in pragmatics have been linked to theory of mind deficiencies (Happé, 1993). Individuals with ASD are reported to produce fewer mental state terms in their narratives (Baron-Cohen et al., 1986). In particular, TagerFlusberg (2000) underlines their difficulties in using cognition verbs (e.g., think, know, guess) that specifically require theory of mind abilities.

The present article reports an exploratory study that seeks to examine the connections between gaze patterns and communicative competencies. It is part of a larger research program that aims at engineering innovative technologies for investigating gazing patterns in ASD. The method presented in this paper could be applied to various features of social communication, such as language fluency, mental state attribution or emotional expression. Here, we illustrate the potential of this method for revealing associations between gaze patterns and pragmatic competencies. More precisely, the method was used to examine correlations between visual fixations on faces and the production of cognition verbs.

A major pitfall of eye-tracking is that it merely measures focal vision and therefore cannot account for attention that is allotted to the periphery of the visual field. This distinction appears to be especially crucial in ASD, given the clinical reports of individuals with ASD having a greater tendency to rely on peripheral vision (Mottron et al., 2007; Noris et al., 2012). To address this possible confound, when using static stimuli, Spezio et al. (2007) employed the bubble paradigm (Gosselin and Schyns, 2001). In this paradigm, participants are shown series of masked images of a face where only randomly selected portions (bubbles) are visible. The areas of the face that contribute the most to emotion recognition are then computed based on performance obtained with the different masks. This procedure can hardly be directly transposed to dynamic stimuli, as the number of masks would increase exponentially with the number of video frames. We propose an alternative method where the mask is contingent on the gaze orientation: The visible area moves in real time with the focal position of the participant on the screen. This creates a gaze-contingent viewing window that reduces possible reliance on peripheral vision and should hence enhance the congruence between visual attention and focal vision as measured with eye-tracking. In a previous study using this gazecontingent window, participants with ASD viewed realistic animations of expressive virtual humans (Grynszpan et al., 2012a). In the present study, we sought to test a context closer to real life settings, by showing videos of real life social interactions that participants had to subsequently describe. We computed correlations with cognition verbs production, first, in the normal vision condition and, second, using the gaze-contingent viewing window. Those two conditions were compared in two pilot experiments, one with typical individuals and the other with individuals having ASD. These experiments were meant to provide preliminary observations regarding the potential of the proposed method.

\section{METHOD PARTICIPANTS}

In experiment 1 , we tested the feasibility of the method with a normative group of 11 typical adults ( 3 females 8 males), ranging from 24 to 40 years with a mean age of 31.82 [SD $=5.65]$. In this pilot study, our goal was to examine the visual strategies employed by the participants who used the gaze-contingent viewing window and not to compare typical controls with individuals having ASD; therefore, in these preliminary experiments, we did not seek to match the normative group of experiment 1 with the ASD group of experiment 2.

Experiment 2 included 11 participants ( 2 females 9 males) diagnosed with autism by psychiatrists using the DSM-IV R diagnostic criteria. The Autism Diagnostic Interview-Revised (ADI-R; Lord et al., 1994) was used to confirm the diagnosis. Participants' mean score on the Raven's Progressive Matrices (Raven and Court, 1986) was 47.03 [ $S D=10.01]$. Their mean Verbal Intelligence Quotient assessed with the Wechsler Adult Intelligence Scale, 3rd edition (Wechsler, 1997), was 88.91 [SD $=15.33$ ]. The group was thus considered high functioning. Their age ranged from 17 to 31 with a mean of $21.36[S D=4.41]$. This research was prospectively reviewed and approved by the regional ethics committee of Tours, France. An informed consent was obtained from each participant. In addition, parents' consents were obtained for minor participants.

\section{PROCEDURE}

The same design was used in experiment 1 and 2. It was composed of an initial normal vision condition followed by a condition using the gaze-contingent viewing window. In the two conditions, participants watched a 2 min video on a 19 inches computer screen that was positioned above a remote eye-tracker (model EYE-TRAC 6 Desktop from Applied Science Laboratories). In the gaze-contingent viewing window condition, the entire graphic display was blurred (using Gaussian smoothing), except for a window centered on the focal point of the participant that moved in real time with her/his gaze (Figures 1, 2). This viewing window was a rectangle with rounded angles measuring $200 \times 80$ pixels, which amounted to visual angles of $6^{\circ} \times 2^{\circ} 22^{\prime}$, therefore covering the fovea visual region in the horizontal direction. The size of this window was determined so that it could at least encompass the two eyes of any face shown in the video. Further technical details are available in Grynszpan et al. (2012a). Two videos were used, one in each condition. They were randomly counterbalanced across participants. Both were movie extracts displaying a social interaction where two protagonists were acting hypocritically toward a third one. Their behavior was contradicting their speech, thus yielding a comical effect. Visual attention to facial expressions was essential for understanding these 


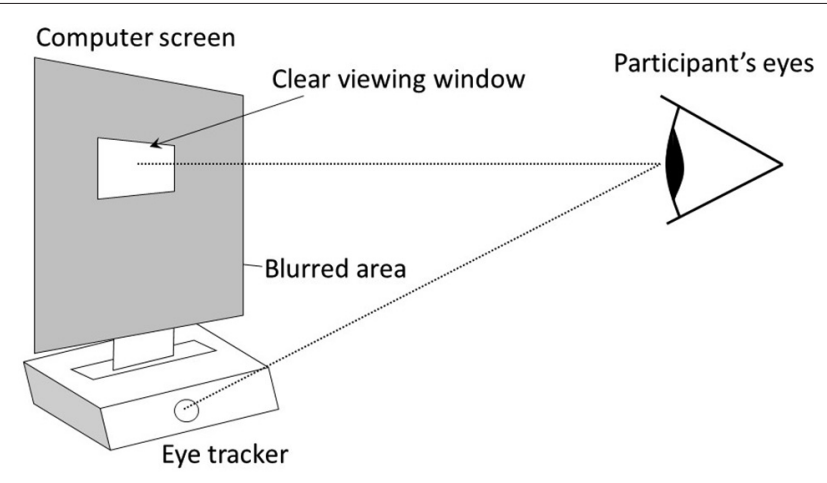

FIGURE 1 | The graphic display is entirely blurred except for a window centered on the focal point of the participant, which is detected in real time using an eye-tracker (model EYE-TRAC 6 Desktop from Applied Science Laboratories, $50 \mathbf{~ H z}$ sampling rate). The eye-tracker remotely measured gaze orientation, without constraining head movements or requiring a helmet.

two movie extracts. In one video $^{1}$, a woman and a man were greeting a neighbor and praising the dish that he prepared, yet their faces and attitudes clearly showed that they were disgusted. In the other video ${ }^{2}$, two men were lauding the dance performance of a woman, although their non-verbal behaviors showed contempt.

After each movie extract, participants were asked to describe what had happened. Their answers were recorded and analyzed by two independent judges who computed the ratio of cognition verbs (e.g., think, believe, know). To calculate this ratio, the number of cognition verbs in each participant's answer was divided by the total number of verbs employed. The concordance correlation coefficient ( $\operatorname{Lin}, 1989)$ between judges was $\rho_{c}=0.91$. To analyze eye-tracking data, rectangular Areas Of Interest (AOI) were defined around the faces of the protagonists. We used a software prototype developed for a previous study (Grynszpan et al., 2012a) to adjust the position of the AOI on video frames so that they would remain centered on the faces throughout the movie extracts. A proprietary algorithm of Applied Science Laboratories was used to compute fixations on the basis of clusters of Points-Of-Gaze (POG) remaining for at least $100 \mathrm{~ms}$ in $1^{\circ}$ of visual angle. Two gaze variables are considered here: the total fixation time on faces, that is, the sum of the fixation durations; and the average fixation duration on faces. In experiment 2, the ADI-R Reciprocal Social Interaction sub-scores and the Childhood Autism Rating Scale (CARS; Schopler et al., 1980) were used as measures of social disability. Although the CARS was originally meant for children, it is also recommended for use in adults (Ozonoff et al., 2005). The ADI-R and the CARS are instruments designed to assist clinicians in diagnosing ASD. The ADI-R is a semi-structured interview of parents or caregivers that is used to retrieve information on the current behavior and developmental history of the individual. The CARS

\footnotetext{
${ }^{1}$ Taken from the film "Le père Noël est une ordure", directed by Jean-Marie Poiré in 1982.

${ }^{2}$ Taken from the movie "Podium", directed by Yann Moix in 2004.
}

is a rating scale based on the observation of the individual's behavior.

\section{RESULTS}

The data analyses were carried out with Statistica software. ${ }^{3}$ For the two experiments, we first verified whether the introduction of the gaze-contingent viewing window altered visual exploration and narrative performance by calculating Student's $t$-tests that compared the two viewing conditions. We then computed Pearson's correlation coefficients between the ratio of cognition verbs and the gaze variables.

In experiment 1 , the data of the normative group showed differences in visual patterns between the two viewing conditions. The total fixation time on faces was higher in the gaze-contingent viewing window condition than in the normal vision condition $\left[t_{(10)}=2.33, p=0.042\right]$. The average duration of fixations on faces was also higher in the gazecontingent viewing window condition compared with the normal vision condition $\left[t_{(10)}=5.70, p<0.001\right]$. The ratio of cognition verbs did not differ significantly between the two viewing conditions. We did not find any correlations between any of these three variables. Indeed, the ratio of cognition verbs did not correlate with the total fixation time on faces [normal vision condition: $r=0.13 p=0.71$; gaze-contingent viewing window condition: $r=0.40 p=0.23$ ], nor did it with the average fixation duration [normal vision condition: $r=0.01$ $p=0.98$; gaze-contingent viewing window condition: $r=0.27$ $p=0.42]$.

In experiment 2 , no significant differences were found between the two viewing conditions on any of the measures, that is, the ratio of cognition verbs $\left[t_{(10)}=0.41, p=0.69\right]$, the total fixation time on faces $\left[t_{(10)}=0.76, p=0.46\right]$ and the average fixation duration on faces $\left[t_{(10)}=0.48, p=0.64\right]$. Contrasting with the normative group in experiment 1 , the total fixation time on faces correlated with the ratio of cognition verbs in the gaze-contingent viewing window condition $[r=$ $0.70, p=0.017]$. The average fixation duration on faces correlated with the ratio of cognition verbs in the normal vision condition $[r=0.60, p=0.05]$ and in the gaze-contingent viewing window condition $[r=0.87, p<0.001]$. In the latter condition, the ratio of cognition verbs correlated negatively with the CARS scores $[r=-0.66, p=0.03]$ and the average duration of fixations on faces correlated negatively with CARS scores $[r=-0.76, p=0.006]$ and with the ADI-R subscores in the Reciprocal Social Interaction domain $[r=-0.65$, $p=0.031]$.

\section{DISCUSSION}

Studies using eye-tracking techniques to analyze gaze exploration in ASD have been mostly involved in the description of facial regions of interests and their link with social impairments. The goal of the present study was to pinpoint more precise components of social impairments, by exploring the potential relationship between gaze exploration of faces and inference of mental states in others. Indeed, inferring mental states is often facilitated

\footnotetext{
${ }^{3}$ www.statsoft.com
} 


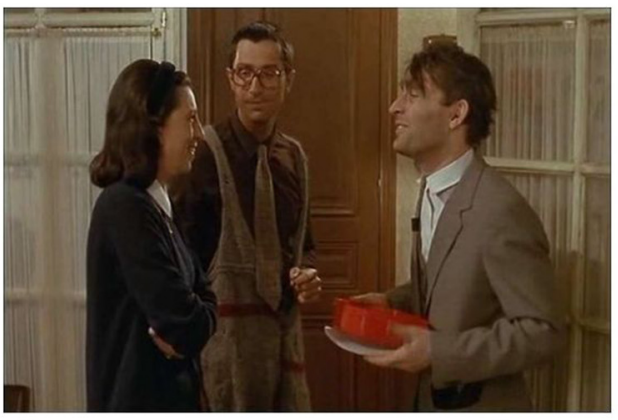

A

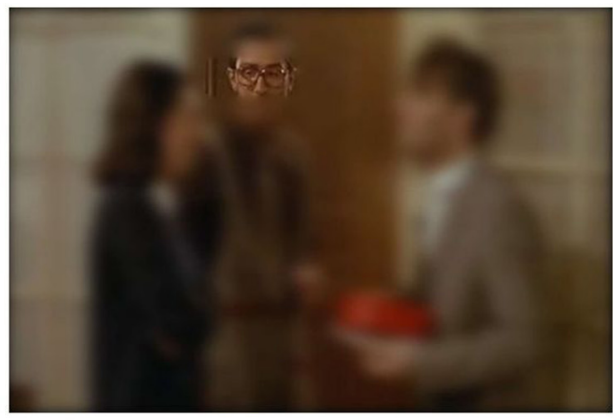

B

FIGURE 2 | Sample screen shots of an extract of the movie "Le père Noël est une ordure" (directed by Jean-Marie Poiré in 1982) that was used as a stimulus $(A)$ in the normal vision condition; $(B)$ in the gaze-contingent viewing window condition.

by deciphering facial expressions. Cognition verbs were used here as indices of mental states processing. Results of experiment 2 show a positive correlation between gaze variables and cognition verbs in participants with ASD. In other words, the more they were attentive to the dynamics of the facial expressions, the more they would use cognition verbs as if the latter were directly derived from the former. This suggests that their mentalistic insight depends predominantly on a face reading strategy whereby mental states are mapped onto behavioral changes perceived on the face. Interestingly, the severity of autism, as assessed by the CARS and the ADI-R sub-scores in the Reciprocal Social Interaction domain, appeared to be related to poor gaze exploration of faces and poor production of mental state terms.

In experiment 1 , the normative group of typical participants modulated their visual behavior to adapt to the gaze-contingent viewing window by increasing their fixation durations on faces. This is consistent with our previous findings (Grynszpan et al., $2012 b)$. The reason why fixation durations on faces did not correlate with the ratio of cognitive verbs for typical participants could conceivably be explained by the fact that their social insight is not solely dependent on facial expressions. In experiment 2, we did not observe an adaptive change in the visual exploration strategies of the ASD group. Although we refrained from comparing groups here, such an alteration in ASD of the typical modulation effect on gaze patterns induced by the viewing window has already been addressed in a previous publication (Grynszpan et al., 2012a). The lack of change in fixation times on faces for ASD participants suggests that they relied on visual strategies when using the gaze-contingent viewing window that were similar to those used in the normal viewing condition. The outcomes derived from the gaze-contingent viewing window should therefore be indicative of their visual exploration in more natural settings.

Recent studies have attempted to tackle discrepancies found in eye-tracking experiments, by devising sophisticated analyses based on the distance between the gaze position of ASD participants and typical gaze patterns (Nakano et al., 2010) or pre-defined targets in the video material (Falck-Ytter et al., 2013). To our knowledge, none of these approaches took into account the possible bias of peripheral vision. Despite anecdotal accounts that individuals with ASD tend to rely more frequently on their peripheral vision than typical peers, especially during social interactions (Bogdashina, 2003; Williams, 2007), there has been relatively little research exploring this issue (Mottron et al., 2007; Grubb et al., 2013; Milne et al., 2013). Most eye-tracking studies focus on central vision, based on the assumption that it matches visual attention. However, detecting that the focal point of gaze is on the mouth does not prevent attention from being directed to the eyes and vice-versa. Such discrepancies could be even more critical in ASD, given the accounts of peculiar use of peripheral vision. The gaze-contingent viewing window method proposed here is meant to reduce this possible confound and indeed, our results showed strong significant correlations with measures of social disability for fixations on the face without having to distinguish between the eyes and mouth regions.

Most eye-tracking studies used the total fixation time on a given $\mathrm{AOI}$ as the main outcome measure (Boraston and Blakemore, 2007). Although the assumption that fixation times on a given detail can be summed makes sense for static stimuli, it is less obvious for dynamic stimuli where the information conveyed by the face changes from one fixation to another. The average fixation duration seems more relevant in this regard as it yields an indication of how long a continuous expressive facial motion is attended to by the participant. In experiment 2, this latter measure was strongly associated with the production of cognition verbs in the normal vision condition and when the viewing window was used.

It should be noted that even with the limited sample of participants with ASD used in experiment 2, the method we propose revealed strong significant associations between mental state attribution abilities, social competence and visual scanning of faces. The participants in our sample were all adults on the higher range of IQ scores. So the present findings cannot be generalized to the entire spectrum. The gaze-contingent viewing window method could reveal alternative strategies when applied to different sub-groups across the spectrum, shedding light on their distinctive cognitive functioning.

The gaze-contingent system that we presented here seems useful to examine visual exploration of social scenes. However, the role of gaze in social communication extends beyond purely 
perceptual functions. It can also assume an active role in faceto-face interactions as for instance in joint attention situations, where it can be used to orient a partner's attention towards an object of interest (Emery, 2000). The development course of ASD is considered to be strongly associated with impairments in joint attention (Charman, 2003). Gaze-contingent displays could be employed to study joint attention. We designed a platform that displays a virtual human character whose gaze orientation is controlled by an eye-tracking device (Courgeon et al., 2014). It can thus be used to simulate joint attention in systematic and controlled conditions that approach naturalistic situations. Our future work will seek to evaluate the potential of this platform for the study of joint attention in the typical population and for individuals with ASD.

\section{ACKNOWLEDGMENTS}

This work was supported by grants from La Fondation de France with La Fondation Adrienne et Pierre Sommer (Project \#2007 005874) and La Fondation Orange (Project \#71/2012). We are particularly thoughtful of Noëlle Carbonell, one of the project's initiator, who passed away before the project was completed. We are very thankful to Dr. Jacques Constant, Florence Le Barillier and all the staff and students of "La Maison pour les personnes autistes" in Chartres. We thank Jérôme Simonin, Pauline Bailleul and Daniel Gepner for their assistance in data collection and technical development.

\section{REFERENCES}

Baron-Cohen, S., Leslie, A. M., and Frith, U. (1986). Mechanical, behavioural and intentional understanding of picture stories in autistic children. Br. J. Dev. Psychol. 4, 113-125. doi: 10.1111/j.2044-835x.1986.tb01003.x

Bogdashina, O. (2003). Sensory Perceptual Issues in Autism and Asperger Syndrome: Different Sensory Experiences, Different Perceptual Worlds. London: Jessica Kingsley Publishers.

Boraston, Z., and Blakemore, S. J. (2007). The application of eye-tracking technology in the study of autism. J. Physiol. 581, 893-898. doi: 10.1113/jphysiol.2007. 133587

Charman, T. (2003). Why is joint attention a pivotal skill in autism? Philos. Trans. R. Soc. Lond. B Biol. Sci. 358, 315-324. doi: 10.1098/rstb.2002.1199

Chawarska, K., Macari, S., and Shic, F. (2013). Decreased spontaneous attention to social scenes in 6-month-old infants later diagnosed with autism spectrum disorders. Biol. Psychiatry 74, 195-203. doi: 10.1016/j.biopsych.2012.11.022

Corden, B., Chilvers, R., and Skuse, D. (2008). Avoidance of emotionally arousing stimuli predicts social-perceptual impairment in Asperger's syndrome. Neuropsychologia 46, 137-147. doi: 10.1016/j.neuropsychologia.2007.08.005

Courgeon, M., Rautureau, G., Martin, J.-C., and Grynszpan, O. (2014). Joint attention simulation using eye-tracking and virtual humans. IEEE Trans. Affect. Comput. 5, 238-250. doi: 10.1109/taffc.2014.2335740

Dalton, K. M., Nacewicz, B. M., Johnstone, T., Schaefer, H. S., Gernsbacher, M. A., Goldsmith, H. H., et al. (2005). Gaze fixation and the neural circuitry of face processing in autism. Nat. Neurosci. 8, 519-526. doi: 10.1038/nn1421

Emery, N. J. (2000). The eyes have it: the neuroethology, function and evolution of social gaze. Neurosci. Biobehav. Rev. 24, 581-604. doi: 10.1016/s01497634(00)00025-7

Falck-Ytter, T., von Hofsten, C., Gillberg, C., and Fernell, E. (2013). Visualization and analysis of eye movement data from children with typical and atypical development. J. Autism Dev. Disord. 43, 2249-2258. doi: 10.1007/s10803-0131776-0

Fletcher-Watson, S., Leekam, S. R., Benson, V., Frank, M. C., and Findlay, J. M. (2009). Eye-movements reveal attention to social information in autism spectrum disorder. Neuropsychologia 47, 248-257. doi: 10.1016/j.neuropsychologia. 2008.07.016
Gosselin, F., and Schyns, P. G. (2001). Bubbles: a technique to reveal the use of information in recognition tasks. Vision Res. 41, 2261-2271. doi: 10.1016/s00426989(01)00097-9

Grubb, M. A., Behrmann, M., Egan, R., Minshew, N. J., Heeger, D. J., and Carrasco, M. (2013). Exogenous spatial attention: evidence for intact functioning in adults with autism spectrum disorder. J. Vis. 13:9. doi: 10.1167/13.14.9

Grynszpan, O., Martin, J., and Nadel, J. (2008). Multimedia interfaces for users with high functioning autism: an empirical investigation. Int. J. Hum. Comput. Stud. 66, 628-639. doi: 10.1016/j.ijhcs.2008.04.001

Grynszpan, O., Nadel, J., Martin, J.-C., Simonin, J., Bailleul, P., Wang, Y., et al. (2012a). Self-monitoring of gaze in high functioning autism. J. Autism Dev. Disord. 42, 1642-1650. doi: 10.1007/s10803-011-1404-9

Grynszpan, O., Simonin, J., Martin, J.-C., and Nadel, J. (2012b). Investigating social gaze as an action-perception online performance. Front. Hum. Neurosci. 6:94. doi: 10.3389/fnhum.2012.00094

Grynszpan, O., Weiss, P. L., Perez-Diaz, F., and Gal, E. (2014). Innovative technology-based interventions for autism spectrum disorders: a meta-analysis. Autism 18, 346-361. doi: 10.1177/1362361313476767

Happé, F. G. (1993). Communicative competence and theory of mind in autism: a test of relevance theory. Cognition 48, 101-119. doi: 10.1016/00100277(93)90026-r

Jones, W., Carr, K., and Klin, A. (2008). Absence of preferential looking to the eyes of approaching adults predicts level of social disability in 2-year-old toddlers with autism spectrum disorder. Arch. Gen. Psychiatry 65, 946-954. doi: 10. 1001/archpsyc.65.8.946

Klin, A., Jones, W., Schultz, R., Volkmar, F., and Cohen, D. (2002a). Defining and quantifying the social phenotype in autism. Am. J. Psychiatry 159, 895-908. doi: 10.1176/appi.ajp.159.6.895

Klin, A., Jones, W., Schultz, R., Volkmar, F., and Cohen, D. (2002b). Visual fixation patterns during viewing of naturalistic social situations as predictors of social competence in individuals with autism. Arch. Gen. Psychiatry 59, 809-816. doi: 10.1001/archpsyc.59.9.809

Lin, L. I.-K. (1989). A concordance correlation coefficient to evaluate reproducibility. Biometrics 45, 255-268. doi: 10.2307/2532051

Lord, C., Rutter, M., and Le Couteur, A. (1994). Autism diagnostic interviewrevised: a revised version of a diagnostic interview for caregivers of individuals with possible pervasive developmental disorders. J. Autism Dev. Disord. 24, 659685. doi: 10.1007/bf02172145

Magrelli, S., Jermann, P., Noris, B., Ansermet, F., Hentsch, F., Nadel, J., et al. (2013). Social orienting of children with autism to facial expressions and speech: a study with a wearable eye-tracker in naturalistic settings. Front. Psychol. 4:840. doi: 10. 3389/fpsyg.2013.00840

Milne, E., Scope, A., Griffiths, H., Codina, C., and Buckley, D. (2013). Brief report: preliminary evidence of reduced sensitivity in the peripheral visual field of adolescents with autistic spectrum disorder. J. Autism Dev. Disord. 43, 19761982. doi: 10.1007/s10803-012-1730-6

Mottron, L., Mineau, S., Martel, G., Bernier, C. S.-C., Berthiaume, C., Dawson, M., et al. (2007). Lateral glances toward moving stimuli among young children with autism: early regulation of locally oriented perception? Dev. Psychopathol. 19, 23-36. doi: 10.1017/s0954579407070022

Nakano, T., Tanaka, K., Endo, Y., Yamane, Y., Yamamoto, T., Nakano, Y., et al. (2010). Atypical gaze patterns in children and adults with autism spectrum disorders dissociated from developmental changes in gaze behaviour. Proc. Biol. Sci. 277, 2935-2943. doi: 10.1098/rspb.2010. 0587

Norbury, C. F., Brock, J., Cragg, L., Einav, S., Griffiths, H., and Nation, K. (2009). Eye-movement patterns are associated with communicative competence in autistic spectrum disorders. J. Child. Psychol. Psychiatry 50, 834-842. doi: 10. 1111/j.1469-7610.2009.02073.x

Noris, B., Nadel, J., Barker, M., Hadjikhani, N., and Billard, A. (2012). Investigating gaze of children with ASD in naturalistic settings. PLoS One 7:e44144. doi: 10. 1371/journal.pone.0044144

Ozonoff, S., Goodlin-Jones, B. L., and Solomon, M. (2005). Evidence-based assessment of autism spectrum disorders in children and adolescents. J. Clin. Child Adolesc. Psychol. 34, 523-540. doi: 10.1207/s15374424jccp 3403_8

Pelphrey, K. A., Sasson, N. J., Reznick, J. S., Paul, G., Goldman, B. D., and Piven, J. (2002). Visual scanning of faces in autism. J. Autism Dev. Disord. 32, 249-261. doi: 10.1023/A:1016374617369 
Picard, R. W. (2000). Affective Computing. Cambridge: MIT Press.

Raven, J. C., and Court, J. H. (1986). Raven's Progressive Matrices and Raven's Coloured Matrices. London: HK Lewis.

Riby, D., and Hancock, P. J. (2009). Looking at movies and cartoons: eye-tracking evidence from Williams syndrome and autism. J. Intellect. Disabil. Res. 53, 169181. doi: 10.1111/j.1365-2788.2008.01142.x

Rice, K., Moriuchi, J. M., Jones, W., and Klin, A. (2012). Parsing heterogeneity in autism spectrum disorders: visual scanning of dynamic social scenes in school-aged children. J. Am. Acad. Child Adolesc. Psychiatry 51, 238-248. doi: 10. 1016/j.jaac.2011.12.017

Schopler, E., Reichler, R. J., DeVellis, R. F., and Daly, K. (1980). Toward objective classification of childhood autism: Childhood Autism Rating Scale (CARS). J. Autism Dev. Disord. 10, 91-103.

Speer, L. L., Cook, A. E., McMahon, W. M., and Clark, E. (2007). Face processing in children with autism: effects of stimulus contents and type. Autism 11, 265-277. doi: 10.1177/1362361307076925

Spezio, M. L., Adolphs, R., Hurley, R., and Piven, J. (2007). Abnormal use of facial information in high-functioning autism. J. Autism Dev. Disord. 37, 929-939. doi: 10.1007/s10803-006-0232-9

Tager-Flusberg, H. (2000). "Language and understanding minds: connections in autism," in Understanding Other Minds, eds S. Baron-Cohen, H. Tager-Flusberg and D. J. Cohen 2nd Edn. (New York: Oxford University Press), 124-149.

van der Geest, J. N., Kemner, C., Verbaten, M. N., and van Engeland, H. (2002). Gaze behavior of children with pervasive developmental disorder toward human faces: a fixation time study. J. Child. Psychol. Psychiatry 43, 669-678. doi: 10. 1111/1469-7610.00055 von Hofsten, C., Uhlig, H., Adell, M., and Kochukhova, O. (2009). How children with autism look at events. Res. Autism Spectr. Disord. 3, 556-569. doi: 10.1016/j. rasd.2008.12.003

Wechsler, D. (1997). Wechsler Adult Intelligence Scale-III (WAIS-III). San Antonio, TX: The Psychological Corporation.

Williams, D. (2007). Nobody nowhere: the extraordinary autobiography of an autistic [text]. Available online at: http://litmed.med.nyu.edu/Annotation? action=view\&annid=12701. Accessed on October 9, 2014.

Conflict of Interest Statement: The authors declare that the research was conducted in the absence of any commercial or financial relationships that could be construed as a potential conflict of interest.

Received: 06 June 2014; accepted: 22 December 2014; published online: 14 January 2015.

Citation: Grynszpan $\mathrm{O}$ and Nadel J (2015) An eye-tracking method to reveal the link between gazing patterns and pragmatic abilities in high functioning autism spectrum disorders. Front. Hum. Neurosci. 8:1067. doi: 10.3389/fnhum.2014.01067

This article was submitted to the journal Frontiers in Human Neuroscience.

Copyright (c) 2015 Grynszpan and Nadel. This is an open-access article distributed under the terms of the Creative Commons Attribution License (CC BY). The use, distribution and reproduction in other forums is permitted, provided the original author(s) or licensor are credited and that the original publication in this journal is cited, in accordance with accepted academic practice. No use, distribution or reproduction is permitted which does not comply with these terms. 